\title{
Quasi-exactly solvable Lie algebras of differential operators in two complex variables
}

\author{
Artemio González-Lópezł, Niky Kamranł and Peter J Olver§ \\ † Departamento de Métodos Matemáticos, Universidad Complutense, 28040 Madrid, Spain \\ ¥Department of Mathematics, McGill University, Montréal, Québec, Canada H3A 2K6 \\ $\$$ School of Mathematics, University of Minnesota, Minneapolis, MN 55455, USA
}

Received 23 April 1991

Abstract. We completely classify all 'quasi-exactly solvable' Lie algebras of first-order differential operators in two complex variables. Applications to quasi-exactly solvable quantum problems are indicated.

\section{Introduction}

Quantum mechanical problems can roughly be divided into two classes. The first class consists of the few 'exactly solvable' models, such as the harmonic oscillator, whose complete spectrum can be exactly analysed using algebraic or group-theoretical techniques. The second class then lumps together all other quantum systems, whose spectrum cannot be explicitly calculated, but can only, at best, be approximated numerically. Recently, work of Turbiner and collaborators $[12-14]$ has pointed out the existence of a new, intermediate class of problems, named 'quasi-exactly solvable', for which some non-empty part of the (point) spectrum can be exactly characterized, while the remainder of the spectrum is, as in the second class, of non-explicit form. The theoretical foundation underlying these quasi-exactly solvable problems is the existence of a hidden symmetry group with a finite-dimensional module of wavefunctions. Specifically, we will call a differential operator Lie algebraic, as in [8], if it can be written as a bilinear combination of first-order differential operators which generate a finite-dimensional Lie algebra. The problem of classifying Lie algebraic Hamiltonian operators was posed by Levine [9] in the context of molecular dynamics, since such Hamiltonians are amenable to the so-called algebraic approach to scattering theory [2]. Furthermore, we will call a finite-dimensional Lie algebra of (first order) differential operators quasi-exactly solvable if it has a finite-dimensional representation on some subspace of the space of smooth functions. (Since our results are local, we will leave aside questions of whether the functions in the representation space are $L^{2}$ integrable, and therefore genuine wavefunctions.) Therefore, in this terminology, a quasi-exactly solvable differential operator is a Lie algebraic differential operator corresponding to a quasi-exactly solvable Lie algebra of differential operators, the "hidden symmetry algebra'. In this case, any finite-dimensional representation space associated with the hidden symmetry algebra is invariant under the given differential operator, and hence that part of the spectrum can be computed algebraically via a finite matrix eigenvalue problem. See [13] for the details of this method.

For one-dimensional problems, complete results are known. Let $p$ denote the derivative operator or (ignoring factors of $\hbar$ and $i$ ) momentum, $p=\mathrm{d} / \mathrm{d} x$. According 
to [8], a complete list of finite-dimensional Lie algebras of first-order differential operators in one (real or complex) variable, up to equivalence, is provided by the one-parameter family of Lie algebras

$$
\mathrm{g}_{c}=\operatorname{span}\left\{p, x p, x^{2} p+c x, 1\right\}
$$

and their subalgebras. Here $c$ is a (real or complex) parameter; see [8] for a more detailed statement of this result. Turbiner [14] has proved that the condition that the Lie algebra $\mathbf{g}_{c}$ be quasi-exactly solvable, i.e. that it possess a finite-dimensional module of functions $f(x)$, requires that the parameter $c$ satisfy a 'quantization' condition $c=-n$, where $n$ is a non-negative integer. Therefore, a complete list of quasi-exactly solvable Lie algebras in one dimension is provided by the algebras $\mathrm{g}_{-n}, 0 \leqslant n \in \mathbb{Z}$, and their subalgebras. By taking bilinear combinations of the generators of one of these algebras, one obtains a complete list of quasi-exactly solvable Schrödinger operators in one dimension $[8,14]$. The associated quantum potentials include many of interest, such as the harmonic oscillator, one-soliton (Pöschl-Teller), Morse and elliptic function potentials.

The purpose of this paper is to extend these classification results to Lie algebras of first-order differential operators in two complex variables $x, y$. (The real case would be similar, cf [5], but slightly more involved.) In an earlier paper [3] (see also [4] for a more detailed account) we completely classified all finite-dimensional Lie algebras of first-order differential operators in two complex variables. Therefore, we need only determine those Lie algebras which satisfy the quasi-exactly solvable condition, i.e. possess a finite-dimensional module of smooth functions. Employing the general techniques from our earlier paper, we find that this problem can be completely resolved. Surprisingly, all the 'cohomology' parameters (an example being the parameter $c$ in the above one-dimensional Lie algebras $\mathbf{g}_{c}$ ) which enter into our classification of Lie algebras of first-order differential operators must, as in the one-dimensional case, satisfy a similar quantization condition that they can assume only a discrete set of values. We do not know at present why this quantization condition always arises, nor whether it will hold in general, e.g. for Lie algebras of differential operators in three variables. This phenomenon of the 'quantization of cohomology' is an intriguing problem that deserves more investigation.

Every bilinear combination of the generators of a quasi-exactly solvable Lie algebra of first-order differential operators produces a quasi-exactly solvable second-order differential operator, although the operator may not be written in a convenient form, or coordinate system, e.g. a Schrödinger operator of the form $\Delta+V$, with $\Delta$ denoting the Laplace-Beltrami operator on a (possibly curved) space and $V(x)$ a scalar potential. In fact, once the dimension is larger than one, not every second-order differential operator is equivalent to such a Schrödinger operator, although complete necessary and sufficient conditions for equivalence are not hard to find. Nevertheless, it appears to be a rather complicated problem to determine which potentials over a given curved space can be written in quasi-exactly solvable form. Shifman and Turbiner [13] have produced several special, intriguing examples of quasi-exactly solvable Schrödinger operators in two variables, but the general theory awaits a more complete development.

We begin by recalling some general facts about the classification problem for Lie algebras of first-order differential operators $[3,4]$. In local coordinates $x=\left(x_{1}, \ldots, x_{n}\right)$ on a manifold (open set) $M$, any first-order differential operator takes the form

$$
\mathscr{D}=\sum_{i=1}^{n} g_{i}(x) \frac{\partial}{\partial x_{i}}+f(x)=g(x) \frac{\partial}{\partial x}+f(x)
$$


where $g_{i}, f \in C^{\infty}(M)$ are smooth scalar-valued functions on $M$. Thus $\mathscr{D}$ is the sum of a vector field $\boldsymbol{v}=\boldsymbol{g}(\boldsymbol{x}) \partial / \partial \boldsymbol{x}$, and a multiplication operator $f(\boldsymbol{x})$. The Lie bracket between differential operators is the usual commutator $[\mathscr{D}, \mathscr{C}]=\mathscr{D} \mathscr{C}-\mathscr{E} \mathscr{D}$.

There are two natural classes of coordinate changes which act on the algebra of differential operators. The first class consists of all (invertible) smooth changes of variables: $\tilde{\boldsymbol{x}}=\boldsymbol{\varphi}(\boldsymbol{x})$. The second class are the 'gauge transformations' given by rescalings of the wavefunction by smooth (non-zero) functions $\psi(x)$, which correspondingly rescales a differential operator according to the rule

$$
\overline{\mathscr{D}}=\psi(\boldsymbol{x}) \mathscr{D} \frac{1}{\psi(\boldsymbol{x})} .
$$

We will call two (Lie algebras of) differential operators equivalent if there is a change of variables $\bar{x}=\varphi(x)$ and a scalar-valued function $\psi(x)$ such they are related by (3). Clearly, we need only classify Lie algebras of differential operators up to equivalence.

A finite-dimensional Lie algebra $\mathfrak{g}$ of first-order differential operators has a basis of the form

$$
\boldsymbol{v}_{1}+f_{1}(\boldsymbol{x}), \ldots, v_{r}+f_{r}(\boldsymbol{x}), h_{1}(\boldsymbol{x}), \ldots, h_{m}(\boldsymbol{x}) .
$$

Here $v_{1}, \ldots, v_{r}$ are linearly independent vector fields spanning an $r$-dimensional Lie algebra $\mathfrak{b}$. The functions $h_{1}(x), \ldots, h_{m}(x)$ define multiplication operators, and span a finite-dimensional b-module $m \subset C^{\infty}(M)$ of smooth functions. (By 'b-module' we mean a (finite-dimensional) representation space for the Lie algebra $\mathfrak{h}$, i.e. if $v \in \mathfrak{h}$, and $h(x) \in m$, then $v(h)(x) \in m$ also.) Finally, the functions $f_{i}(x)$ can be interpreted as defining a '1-cochain' for the Lie algebra $\mathfrak{h}$, given as the linear map $F: \mathfrak{h} \rightarrow C^{\infty}(M)$, defined by $\left\langle\boldsymbol{F} ; \boldsymbol{v}_{j}\right\rangle=f_{j}$, and extended to all of $\mathfrak{h}$ by linearity. Actually, since we can add in any constant coefficient linear combination of the $h_{i}$ to the $f_{j}$ without changing the Lie algebra $\mathrm{g}$, we should interpret the $f_{j}$ as lying in the quotient module $C^{\infty}(M) / m$, and hence regard $F$ as a $C^{\infty}(M) / m$-valued cochain. Recall that the coboundary of a 1-cochain $\boldsymbol{F}$ is defined as the bilinear map $\delta_{1} \boldsymbol{F}: \mathfrak{h} \times \mathfrak{h} \rightarrow C^{\infty}(\boldsymbol{M}) / \mathrm{m}$ (or 2-cochain) given by the standard formula

$$
\left\langle\delta_{1} F ; v, w\right\rangle \equiv v\langle F ; w\rangle-w\langle F ; v\rangle-\langle F ;[v, w]\rangle \quad \text { for } v, w \in \mathfrak{h} .
$$

(See Jacobson [6] for the necessary facts from Lie algebra cohomology.) The cochain $F$ is called a cocycle if its coboundary $\delta_{1} F=0$ vanishes, meaning that the right-hand side of (5) lies in the module $m$ for each pair of Lie algebra elements $v, w \in h$, which is readily seen to be the same as the condition that the entire collection of differential operators (4) span a Lie algebra, cf [3]. On the other hand, the cocycle $F$ is itself a coboundary, written $F=\delta_{0} \varphi$, if it has the form $\left\langle F ; v_{j}\right\rangle=v_{j}(\varphi)$ for some smooth function $\varphi \in C^{\infty}(M)$. Two such cocycles will differ by a coboundary $\delta_{0} \varphi$ if and only if the corresponding Lie algebras are equivalent under a rescaling of the wavefunction (3) with $\psi=\mathrm{e}^{-\varphi}$. Therefore two cocycles lying in the same cohomology class, in the first Lie algebra cohomology space $H^{1}\left(\mathfrak{h}, C^{\infty}(M) / m\right)=\operatorname{ker} \delta_{1} / \operatorname{Im} \delta_{0}$, will give rise to equivalent Lie algebras of differential operators.

In summary, then, we have the following fundamental characterization of Lie algebras of first-order differential operators $[3,4]$.

Theorem 1. There is a one-to-one correspondence between equivalence classes of finite-dimensional Lie algebras of first-order differential operators on $M$ and equivalence classes of triples $[\mathfrak{h}, m,[\boldsymbol{F}]]$, where:

1. $\mathfrak{h}$ is a finite-dimensional Lie algebra of vector fields; 
2. $m \subset C^{\infty}(M)$ is a finite-dimensional $\mathfrak{h}$-module of functions;

3. [F] is a cohomology class in $H^{1}\left(\mathfrak{h}, C^{\infty}(M) / m\right)$.

Two such triples are equivalent if they are directly mapped to each other by a change of variables $\bar{x}=\varphi(x)$, the cohomology taking care of the rescaling (3).

For example, in the case of the Lie algebras $g_{c}$, given in (1), the corresponding triple consists of the Lie algebra of vector fields $\mathfrak{h}=\operatorname{span}\left\{p, x p, x^{2} p\right\}$, generating the group of linear fractional transformations $\$ \mathbb{I}(2)$. The module $m=\{1\}$ consists of just the constant functions, spanned by the constant function 1. (It can be seen that this is the only non-zero $\mathfrak{h}$-module of functions.) Finally, the cohomology class is represented by the cocycle or linear map $\boldsymbol{F}$ which has the values $\langle\boldsymbol{F} ; p\rangle=0,\langle\boldsymbol{F} ; x p\rangle=0$ and $\left\langle\boldsymbol{F} ; x^{2} p\right\rangle=c x$, on the generators of $\mathfrak{h}$.

According to theorem 1 , there are three basic steps required to classify finitedimensional Lie algebras of first-order differential operators. First, one needs to classify the finite-dimensional Lie algebras of vector fields $\mathfrak{b}$ on the manifold $M$ up to changes of variables. Secondly, for each of these Lie algebras, one needs to classify all possible finite-dimensional $\mathfrak{h}$-modules $m$ of $C^{\infty}$ functions. Finally, for each of the modules $m$, one needs to determine the first cohomology space $H^{\mathfrak{t}}\left(\mathfrak{h}, C^{\infty}(M) / m\right)$. For $M=\mathbb{R}$ this was done in [8]. For $M=\mathbb{C}^{2}$, the first step was effected by Lie [10,11] and the second and third by the present authors $[3,4]$. Finally, for $M=\mathbb{R}^{2}$, only the first step, i.e. classifying finite-dimensional Lie algebras of vector fields, has been completed [5].

Tables 1-3 summarize the classification results of finite-dimensional Lie algebras of differential operators in two complex variables. The derivative operators (momenta) are denoted by $p=\partial / \partial x, q=\partial / \partial y$. Lie's classification of generic finite-dimensional Lie algebras of vector fields on $\mathbb{C}^{2}$ is summarized in table 1 . ('Generic' means that we are avoiding singular points where the dimension of the orbits of the Lie algebra varies, e.g. singularities of vector fields.) The first column provides our identification number for the indicated class of Lie algebras. The second column gives a basis for the algebra, and the third indicates its structure as an abstract Lie algebra. Here, $\mathfrak{h}_{2}=\mathbb{C} \times \mathbb{C}$ denotes the unique solvable two-dimensional Lie algebra, $\propto$ denoting semi-direct product. The last column indicates where the Lie algebra lies in Lie's 'Gruppenregister' [11]. (We have, in a few cases, used different coordinate systems than Lie.) Table 2 gives the different finite-dimensional modules for each of these Lie algebras. Trivial modules, valid for any Lie algebra of vector fields are the zero module $m=0$, which consists of the zero function alone, and that containing just the constant functions, which we write $m=\{1\}$. Note that, if our Lie algebra is spanned by vector fields, then table 2 completes the solution to the problem of classifying quasi-exactly solvable Lie algebras of vector fields in two complex variables. In cases $11,15,23$ and 24 the only non-zero finite-dimensional module consists just of constants, but this can still have interesting consequences for associated quantum Hamiltonians, e.g. the characterization of the ground state. Lie algebras $1,2,3,16,17$ are of little relevance for planar quantum mechanics, as the differential operators which can be expressed as bilinear combinations of their generators are just one-dimensional, in particular can never be elliptic, let alone Laplacian plus potential. However as the analysis is not too complicated, we retain these cases in the subsequent discussion. In table 2, the first column gives the identification number of the Lie algebra considered from table 1 . The second column shows whether the module is necessarily spanned by monomials, i.e. single terms of the indicated form. (In cases 5 and 20, we have monomials unless $\alpha \in \mathbb{Q}^{+}$or $r<\alpha \in \mathbb{Q}^{+}$ are positive rational numbers, respectively.) The third column indicates a typical term 
Table 1. Finite-dimensional Lie algebras of vector fields in the complex plane.

\begin{tabular}{|c|c|c|c|}
\hline & Generators & Structure & Label \\
\hline 1 & $\{p\}$ & C & $E$ \\
\hline 2 & $\{p, x p\}$ & $\mathbf{b}_{2}$ & $C 1$ \\
\hline 3 & $\left\{p, x p, x^{2} p\right\}$ & $\$ 1(2)$ & $C 4$ \\
\hline 4 & $\{p, q\}$ & $\mathbb{C}^{2}$ & $D 1$ \\
\hline 5 & $\{p, q, x p+\alpha y q\} \quad \alpha \neq 0$ & $\mathbb{C} \ltimes \mathbb{C}^{2}$ & $C 8, D 3$ \\
\hline 6 & $\{p, q, x p, y q\}$ & $\mathfrak{h}_{2} \oplus \mathfrak{h}_{2}$ & $C 3$ \\
\hline 7 & $\{p, q, x p-y q, y p, x q\}$ & $\operatorname{sl}(2) \times \mathbb{C}^{2}$ & $A 3$ \\
\hline 8 & $\{p, q, x p, y p, x q, y q\}$ & $\operatorname{gl}(2) \propto \mathbb{C}^{2}$ & $A 2$ \\
\hline 9 & $\left\{p, q, x p, x^{2} p\right\}$ & $\operatorname{gl}(2)$ & $C 5$ \\
\hline 10 & $\left\{p, q, x p, y q, x^{2} p\right\}$ & $\mathbf{s l}(2) \oplus \mathbf{h}_{2}$ & C6 \\
\hline 11 & $\left\{p, q, x p, y q, x^{2} p, y^{2} q\right\}$ & $\mathbf{s l}(2) \oplus \mathbf{s l}(2)$ & $C 7$ \\
\hline 12 & $\left\{p+q, x p+y q, x^{2} p+y^{2} q\right\}$ & $\$ 1(2)$ & $C 9$ \\
\hline 13 & $\left\{p, 2 x p-y q, x^{2} p-x y q\right\}$ & $\$ 1(2)$ & $B \delta 1$ \\
\hline 14 & $\left\{p, x p, y q, x^{2} q-x y q\right\}$ & $\operatorname{gl}(2)$ & $B \delta 2$ \\
\hline 15 & $\left\{p, q, x p, y p, x q, y q, x^{2} p+x y q, x y p+y^{2} q\right\}$ & sl( $(3)$ & $A 1$ \\
\hline 16 & $\left\{\xi_{1}(x) q, \ldots, \xi_{r}(x) q\right\}, \quad r>1$ & $\mathbb{C}^{r}$ & $B \alpha 1$ \\
\hline 17 & $\left\{\xi_{1}(x) q, \ldots, \xi_{r}(x) q, y q\right\}, \quad r>1$ & $\mathbb{C} \times \mathbb{C}^{r}$ & $B \alpha 2$ \\
\hline 18 & $\left\{p, x^{i} \mathrm{e}^{\lambda x} q: 0 \leqslant i \leqslant r_{\lambda}\right\}, \quad \lambda \in \Lambda, \quad r=\Sigma r_{\lambda}$ & $\mathbb{C} \ltimes \mathbb{C}^{r}$ & $B \beta 1, D 2$ \\
\hline 19 & $\left\{p, y q, x^{i} \mathrm{e}^{\lambda x} q: 0 \leqslant i \leqslant r_{\lambda}\right\}, \quad \lambda \in \Lambda, \quad r=\Sigma r_{\lambda}$ & $\mathbb{C}^{2} \times \mathbb{C}^{r}$ & $B \beta 2, C 2$ \\
\hline 20 & $\left\{p, q, x p+\alpha y q, x q, \ldots, x^{r} q\right\}, \quad r \geqslant 1$ & $\mathfrak{h}_{2} \times \mathbb{C}^{r+1}$ & $B \gamma 1,2$ \\
\hline 21 & $\left\{p, q, x q, \ldots, x^{r-i} q, x p+\left(r y+x^{r}\right) q\right\}, \quad r \geqslant 1$ & $\mathfrak{h}_{2} \propto \mathbb{C}^{\prime}$ & $B \gamma 3$ \\
\hline 22 & $\left\{p, q, x p, x q, y q, x^{2} q, \ldots, x^{\prime} q\right\}, \quad r \geqslant 1$ & $\left(\mathbf{h}_{2} \oplus \mathbb{C}\right) \times \mathbb{C}^{r+1}$ & $B \gamma 4$ \\
\hline 23 & $\left\{p, q, 2 x p+r y q, x q, x^{2} p+r x y q, x^{2} q, \ldots, x^{r} q\right\}, \quad r \geqslant 1$ & $\$ 1(2) \times \mathbb{C}^{r+1}$ & $B \delta 3$ \\
\hline 24 & $\left\{p, q, x p, x q, y q, x^{2} p+r x y q, x^{2} q, \ldots, x^{r} q\right\}, \quad r \geqslant 1$ & $\operatorname{gl}(2) \ltimes \mathbb{C}^{r+1}$ & $B \delta 4$ \\
\hline
\end{tabular}

in a basis element for the module; $i, j$ always denote non-negative integers. If the module is not spanned by monomials, then the generators will be certain linear combinations of the indicated monomials. However, in all non-monomial cases, the generators can still be taken to be 'exponentially homogeneous', i.e. only one type of exponential appears in each basis element. The fourth column either indicates ranges of indices which must be included, or, in the case of an arrow, it indicates other indices which must be included if the given one is. For instance, in case 19, if the monomial $x^{i} y^{j} \mathrm{e}^{\mu x}$ belongs to the module, so must the monomials $x^{i-1} y^{j} \mathrm{e}^{\mu x}$ and $x^{i+r_{\lambda}} y^{j-1} \mathrm{e}^{(\mu+\lambda) y}$ (provided $i>0$ and/or $j>0$ ) for each exponent $\lambda$ appearing in the Lie algebra. Cases when the module is not generated by monomials must be treated with a bit of care, as certain coefficients will also appear, $\mathrm{cf}[3]$. In all cases, the arbitrary functions (e.g. the $g(y)$ in cases 1-3) or the exponents (e.g. the $\lambda$ and $\mu$ in case 4) are restricted to belong to a finite set, so that the module is finite-dimensional. Finally, $Q_{n}^{k}$ denotes the ultraspherical polynomial

$$
Q_{n}^{k}(z)=\frac{\mathrm{d}^{2 n-k}}{\mathrm{~d} z^{2 n-k}}\left(z^{2}-1\right)^{n}
$$

which is a multiple of the Gegenbauer polynomial $C_{k}^{n-k+(1 / 2)} ; \mathrm{cf}[1]$.

Table 3 describes the cohomology spaces $H^{1}\left(\mathfrak{h}, C^{\infty}(M) / m\right)$ for each of the Lie algebras and corresponding modules appearing in tables 1 and 2 . The first column indicates the dimension of the cohomology space, and the second column gives a representative cocycle $\boldsymbol{F}$ of each non-trivial cohomology class. Only the vector fields which are actually modified are indicated, i.e. in the notation of (4), only the differential 
Table 2. Finite-dimensional modules for Lie algebras of vector fields.

\begin{tabular}{rlll}
\hline & Monomials? & Generators & Rules \\
\hline 1 & No & $x^{i} \mathrm{e}^{\lambda x} \mathrm{~g}(y)$ & $(i, \lambda, g) \rightarrow(i-1, \lambda, g)$ \\
2 & Yes & $x^{i} g(y)$ & $(i, g) \rightarrow(i-1, g)$ \\
3 & Yes & $g(y)$ & \\
4 & No & $x^{i} y^{j} \mathrm{e}^{\lambda x} \mathrm{e}^{\mu y}$ & $(i, j, \lambda, \mu) \rightarrow(i-1, j, \lambda, \mu),(i, j-1, \lambda, \mu)$ \\
5 & $\alpha \in \mathbb{Q}^{+}$ & $x^{i} y^{j}$ & $(i, j) \rightarrow(i-1, j),(i, j-1)$ \\
6 & Yes & $x^{i} y^{j}$ & $(i, j) \rightarrow(i-1, j),(i, j-1)$ \\
7 & Yes & $x^{i} y^{j}$ & $0 \leqslant i+j \leqslant n$ \\
8 & Yes & $x^{i} y^{j}$ & $0 \leqslant i+j \leqslant n$ \\
9 & Yes & $y^{j} \mathrm{e}^{\mu y}$ & $0 \leqslant j \leqslant n_{\mu}$ \\
10 & Yes & $y^{j}$ & $0 \leqslant j \leqslant n$ \\
11 & Yes & 1 & \\
12 & No & $(x-y)^{k-n} Q_{k}^{n}((x+y) /(x-y))$ & $0 \leqslant k \leqslant 2 n$ \\
13 & Yes & $x^{i} y^{n}$ & $0 \leqslant i \leqslant n, n \in S$ \\
14 & Yes & $x^{i} y^{n}$ & $0 \leqslant i \leqslant n, n \in S$ \\
15 & Yes & 1 & \\
16 & No & $y^{j} g(x)$ & $(j, g) \rightarrow\left(j-1, g \cdot \xi_{k}\right)$ \\
17 & Yes & $y^{j} g(x)$ & $(j, g) \rightarrow\left(j-1, g \cdot \xi_{k}\right)$ \\
18 & No & $x^{i} y^{j} \mathrm{e}^{\mu x}$ & $(i, j, \mu) \rightarrow(i-1, j, \mu),\left(i+r_{\lambda}, j-1, \mu+\lambda\right)$ \\
19 & Yes & $x^{i} y^{j} \mathrm{e}^{\mu x}$ & $(i, j, \mu) \rightarrow(i-1, j, \mu),\left(i+r_{\lambda}, j-1, \mu+\lambda\right)$ \\
20 & $r<\alpha \in \mathbb{Q}^{+}$ & $x^{i} y^{j}$ & $(i, j) \rightarrow(i-1, j),(i+r, j-1)$ \\
21 & Yes & $x^{i} y^{j}$ & $(i, j) \rightarrow(i-1, j),(i+r, j-1)$ \\
22 & Yes & $x^{i} y^{j}$ & $(i, j) \rightarrow(i-1, j),(i+r, j-1)$ \\
23 & Yes & 1 & \\
24 & Yes & 1 & \\
\hline & & &
\end{tabular}

operators $\boldsymbol{v}_{i}+f_{i}$ with non-zero $f_{i}=\left\langle\boldsymbol{F} ; \boldsymbol{v}_{i}\right\rangle \neq 0$ are explicitly written down. In case 4 , $\operatorname{div} m=\left\{f_{x}+g_{y} \mid f, g \in m\right\}$. The classes are, in most cases, parametrized by complex numbers $c_{i}$. Some of these cohomology classes, especially cases 16,18 and 20 , are quite complicated to describe, and we refer the reader to $[3,4]$ for a complete discussion. Thus, taken together, tables 1-3 completely solve the probiem of classifying generic finite-dimensional Lie algebras of first-order differential operators in two complex variables.

Example. Consider the Lie algebra of vector fields of type 7, corresponding to the group of affine volume-preserving maps of the plane. When the module $m=\{1\}$ consists of the constants, table 3 shows that the cohomology is one-dimensional. Thus, the corresponding Lie algebras of first-order differential operators are given by

$$
\mathbf{g}_{c}^{(7)}=\operatorname{span}\left\{p, q+2 c x, x p-y q, y p+c y^{2}, x q+c x^{2}, 1\right\}
$$

where $c$ parametrizes the cohomology class. We leave it to the reader to verify that these do define a one-parameter family of inequivalent Lie algebras of differential operators.

Turning to the problem to be addressed here, we must determine which of the Lie algebras from tables 1-3 satisfy the quasi-exactly solvable condition that they admit a non-zero finite-dimensional module $n$ of smooth functions. The results are summarized in table 4 . The rest of our paper will be devoted to a discussion of these results, and indications of proofs for some of the more complicated cases. 
Table 3. Cohomologies for Lie algebras of vector fields.

\begin{tabular}{|c|c|c|c|}
\hline & \multicolumn{2}{|l|}{ Dimension } & Representatives \\
\hline 1 & 0 & & \\
\hline 2 & $\infty$ & & $x p+h(y), \quad h \notin m$ \\
\hline 3 & $\infty$ & & $x p+h(y), \quad x^{2} p+2 x h(y)$ \\
\hline 4 & $\operatorname{dim} m / \operatorname{div} m$ & $<\infty$ & $q+h(x, y), \quad h_{x} \in m, \quad h \neq \psi_{y}$ with $\psi_{x} \in m$ \\
\hline 5 & $0\left(\alpha \notin \mathbf{Q}^{-}\right)$ & 1 or $0\left(\alpha \in \mathbf{Q}^{-}\right)$ & $x p+c y q+c_{1} x^{i} y^{j}$ or $q+c_{1} x^{i} y^{j}$ \\
\hline 6 & $0(m \neq 0)$ & $2(m=0)$ & $x p+c_{1}, \quad y q+c_{2}$ \\
\hline 7 & $0(m \neq\{1\})$, & $1(m=\{1\})$ & $q+2 c_{1} x, \quad y p+c_{1} y^{2}, \quad x q+c_{1} x^{2}$ \\
\hline 8 & $0(m \neq 0)$ & $1(m=0)$ & $x p+c_{1}, \quad y q+c_{1}$ \\
\hline 9 & 1 & & $x p+c_{1}, \quad x^{2} p+2 c_{1} x$ \\
\hline 10 & $1(m \neq 0)$ & $2(m=0)$ & $x p+c_{1}, \quad x^{2} p+2 c_{1} x, \quad y q+c_{2}$ \\
\hline 11 & 2 & & $x p+c_{1}, \quad x^{2} p+2 c_{1} x, \quad y q+c_{2}, \quad y^{2} q+2 c_{2} y$ \\
\hline 12 & 1 & & $x^{2} p+y^{2} q+c_{1}(x-y)$ \\
\hline 13 & 1 & & $x^{2} p-x y q+c_{1} y^{-2}$ \\
\hline 14 & $0(1 \in m)$ & $1(1 \notin m)$ & $x p+c_{1}, \quad y q+2 c_{1}$ \\
\hline 15 & & & $x p+c_{1}, \quad x^{2} p+x y q+3 c_{1} x, \quad y q+c_{1}, \quad x y p+y^{2} q+3 c_{1} y$ \\
\hline 16 & $\infty^{r}+<\infty$ & & $\xi_{i}(x) q+f_{i}(x) y^{j}$ \\
\hline 17 & $\infty$ & & $y q+f(x)$ \\
\hline 18 & $<\infty$ & & $x^{k} \mathrm{e}^{\lambda x} q+c_{i, j}^{\lambda, k} x^{i+k} y^{j} \mathrm{e}^{\lambda x}$ \\
\hline 19 & 1 & & $y q+c_{1} x^{n}$ \\
\hline 20 & $0(\alpha \notin \mathbf{Q})$ & 1 or $0(\alpha \in Q)$ & $x p+\alpha y q+c_{1} x^{i} y^{j}, \quad$ or $x^{k} q+c_{k} x^{i+k} y^{j}, \quad k \geqslant l \geqslant 0$ \\
\hline 21 & $0(m \neq 0)$ & $1(m=0)$ & $x p+\left(r y+x^{r} y\right) q+c_{1}$ \\
\hline 22 & $0(m \neq 0)$ & $2(m=0)$ & $x p+c_{1}, \quad y q+c_{2}$ \\
\hline 23 & $\begin{array}{l}1(r>2) \\
2(r=2)\end{array}$ & & $\begin{array}{l}2 x p+r y q+c_{1}, \quad x^{2} p+r x y q+c_{1} x \\
x p+y q+c_{1}, \quad x q+c_{2}, \quad x^{2} p+2 x y q+2 c_{1} x+2 c_{2} y, \quad x^{2} q+2 c_{2} x\end{array}$ \\
\hline 24 & $1(m \neq 0)$ & $2(m=0)$ & $x p+c_{1}, \quad y q+c_{2}, \quad x^{2} p+r x y q+\left(2 c_{1}+r c_{2}\right) x$ \\
\hline
\end{tabular}

We begin by stating two trivial results which imply that we can always, without loss of generality, take the module $m$ determining the Lie algebra of differential operators to consist of the constant functions, i.e. $m=\{1\}$.

Lemma 2. If $\mathrm{g}$ is a quasi-exactly solvable Lie algebra of differential operators described by the triple $[\mathfrak{b}, m,[F]]$, then either $m=0$ or $m=\{1\}$.

Proof. Suppose $h(x) \in m$ and $0 \neq g(x) \in n$, the finite-dimensional module for $g$. Then since $h$ defines a multiplication operator in $\mathrm{g}$, we must have $h(x) g(x) \in n$ also. Iterating the multiplication operator, we deduce that $h(x)^{n} g(x) \in n$ for any power $n \geqslant 0$. But $n$ is finite-dimensional, so taking $n$ greater than the dimension of $n$, we deduce a linear dependency of the form $\Sigma c_{k} h(x)^{k} g(x)=0$, for constants $c_{k}$. Since $g(x) \neq 0, h$ must satisfy a constant coefficient polynomial equation $\Sigma c_{k} h(x)^{k}=0$. We conclude that $h(x)$ itself is a constant, which proves the lemma.

Lemma 3. Suppose $\mathrm{g}_{0}$ is a quasi-exactly solvable Lie algebra of differential operators described by the triple $[\mathfrak{h}, 0,[F]]$. Then there exists a quasi-exactly solvable Lie algebra of differential operators $\mathbf{g}$ described by a triple $[\mathfrak{h},\{1\},[\tilde{\boldsymbol{F}}]]$, such that $\mathfrak{g}_{0} \subset \mathfrak{g}$ is a subalgebra. Moreover, if $\boldsymbol{n}$ is a finite-dimensional module for $\mathfrak{g}_{0}$, then it is also one for $\mathrm{g}$.

Proof. Indeed, if $F$ is a $C^{\infty}(M)$-valued cocycle representing a non-trivial cohomology class in $H^{1}\left(\mathfrak{h}, C^{\infty}(M)\right)$ when $m=0$, then $\boldsymbol{F}=\tilde{\boldsymbol{F}}+\boldsymbol{F}_{0}$, where $\tilde{\boldsymbol{F}}$ is a $C^{\infty}(M) /\{1\}$-valued 
Table 4. Quasi-exactly solvable Lie algebras of differential operators.

\begin{tabular}{|c|c|c|}
\hline & Quantization condition & Module \\
\hline 1 & 0 & \\
\hline 2 & 0 & \\
\hline 3 & $h=-n / 2, \quad n \geqslant 0$ & $\left\{x^{i} g(y) \mid i \leqslant n, g \in S\right\}$ \\
\hline 4 & 0 & \\
\hline 5 & 0 & \\
\hline 6 & 0 & \\
\hline 7 & 0 & \\
\hline 8 & 0 & \\
\hline 9 & $c_{1}=-n / 2, \quad n \geqslant 0$ & $\left\{x^{i} y^{i} \mathrm{e}^{\mu y} \mid i \leqslant n, j \leqslant m_{\mu}\right\}$ \\
\hline 10 & $c_{1}=-n / 2, \quad n \geqslant 0$ & $\left\{x^{i} y^{j} \mid i \leqslant n, j \leqslant m\right\}$ \\
\hline 11 & $c_{1}=-n / 2, \quad c_{2}=-m / 2, \quad n, m \geqslant 0$ & $\left\{x^{i} y^{j} \mid i \leqslant n, j \leqslant m\right\}$ \\
\hline 12 & $c_{1}=n / 2 \dagger$ & $\left\{(x-y)^{(m+(n / 2)-k)} R_{k}^{m, n}[(x+y) /(x-y)] \mid 0 \leqslant k \leqslant 2 m+n, m \in S\right\}$ \\
\hline 13 & 0 & \\
\hline 14 & 0 & \\
\hline 15 & $c_{1}=-n / 3, \quad n \geqslant 0$ & $\left\{x^{i} y^{j} \mid i+j \leqslant n\right\}$ \\
\hline 16 & 0 & \\
\hline 17 & 0 & \\
\hline 18 & 0 & \\
\hline 19 & 0 & \\
\hline 20 & 0 & \\
\hline 21 & 0 & \\
\hline 22 & 0 & \\
\hline 23 & $c_{1}=-n, \quad n \geqslant 0$ & $\left\{x^{i} y^{j} \mid i+r j \leqslant n\right\}$ \\
\hline 24 & $c_{1}=-n / 2, \quad c_{2}=0, \quad n \geq 0$ & $\left\{x^{i} y^{j} \mid i+r j \leqslant n\right\}$ \\
\hline
\end{tabular}

$\dagger$ In case 12 , there is no positivity restriction on $n$, and $S \subset\{m \mid m \geqslant \max (0,-n)\}$ is a finite set of integers.

cocycle, and so represents a (possibly trivial) cohomology class when $m=\{1\}$, and $F_{0}$ is a constant cochain, i.e. $\left\langle\boldsymbol{F}_{0} ; \boldsymbol{v}\right\rangle=$ constant for all $\boldsymbol{v} \in \mathfrak{h}$. The Lie algebra $g_{0}$, represented by the triple $[\mathfrak{h}, 0,[F]]$, is then easily seen to be a subalgebra of $\mathfrak{g}$, represented by $[\mathfrak{h},\{1\},[\tilde{\boldsymbol{F}}]]$. Indeed, $\mathfrak{g} \simeq \mathfrak{g}_{0}+\{1\}$ is given by appending the constant functions to $\mathfrak{g}_{0}$, which immediately implies the last statement of the lemma.

Therefore, combining lemmas 1 and 2, we see that we can always, without loss of generality, take the Lie algebra of differential operators $g$ to be represented by a triple $[\mathfrak{h},\{1\},[F]]$, i.e. the module $m=\{1\}$ consists of the constant functions. If the cohomology is trivial, $[\boldsymbol{F}]=0$, so that the Lie algebra $g$ is spanned by vector fields and the constant functions, then according to table 2 it always satisfies the quasi-exactly solvable condition, with the associated finite-dimensional modules being explicitly described therein. Therefore, our present task reduces to analysing which non-trivial cohomology classes permit the resulting Lie algebra of differential operators to have some non-zero finite-dimensional module of functions. We will find that, in all cases, either the cohomology must be trivial, or, it must satisfy a 'quantization' condition, that the associated parameters (or arbitrary functions) can only take on a discrete set of values. We now describe in detail some of the calculations required to complete table 4 . Using these as a guide, the interested reader can then complete the analysis for the remaining cases.

Case 3. We begin by presenting an essentially one-dimensional case, primarily as an illustration of the basic method that can be used in the other routine cases that are 
not explicitly discussed. According to table 3 , when $m=\{1\}$, the non-trivial cohomology classes are represented by the Lie algebras

$$
\mathfrak{g}_{h}^{(3)}=\operatorname{span}\left\{p, x p+h(y), x^{2} p+2 x h(y), 1\right\}
$$

where $h$ is an arbitrary function of $y$. Since the $x$-translation vector field $p$ belongs to $\mathbf{g}_{h}^{(3)}$, the associated module $n$ must, according to case 1 of table 2 (cf lemma 1 of [3]) be spanned by functions of the form $r(x, y) \mathrm{e}^{\lambda x}$, where $r$ is a polynomial in $x$. Fixing $\lambda$, suppose

$$
r(x, y)=s_{n}(y) x^{n}+\ldots
$$

is a polynomial of maximal degree in $x$ for which $r(x, y) \mathrm{e}^{\lambda x} \in n$. Then

$$
[x p+h(y)] r(x, y) \mathrm{e}^{\lambda x}=\left[\lambda s_{n}(y) x^{n+1}+\ldots\right] \mathrm{e}^{\lambda x}
$$

must also belong to $n$, which inmediately implies that $n$ is finite-dimensional only if $\lambda=0$, i.e. $n$ is spanned by functions $r(x, y)$ which are polynomial in $x$. Moreover, for $r(x, y)$ as in (7) of maximal degree,

$$
[x p+h(y)] r(x, y)=(n+h(y)) g_{n}(y) x^{n}+\ldots
$$

also belongs to $n$. Iterating this differential operator repeatedly, and employing an argument similar to that in the proof of lemma 1 , we deduce that the module $n$ will be finite-dimensional only if $h(y)=h$ is a constant. Finally,

$$
\left[x^{2} p+2 x h\right] r(x, y)=(n+2 h) g_{n}(y) x^{n+1}+\ldots
$$

also must belong to $n$, which implies that $n+2 h=0$. Hence we deduce the quantization condition $h=-n / 2$, where $n$ is a non-negative integer. The resulting Lie algebras of differential operators are given by

$$
\mathbf{g}_{-n / 2}^{(3)}=\operatorname{span}\left\{p, x p, x^{2} p-n x, 1\right\}
$$

just as in the one-dimensional classification. Moreover, it is not hard to see that any associated finite-dimensional module is spanned by monomials

$$
x^{i} g(y) \quad 0 \leqslant i \leqslant n \quad g(y) \in S
$$

where $S$ denotes a finite set of functions of $y$.

Case 12. Using the change of variables

$$
(x, y) \rightarrow\left(\log (x-y), \frac{x+y}{x-y}\right)
$$

from [3], and the cohomology calculation from table 3, we map the Lie algebra to

$$
\mathfrak{g}_{c}^{(12)}=\operatorname{span}\left\{2 \mathrm{e}^{-x} q, p, \mathrm{e}^{x}\left[2 y p+\left(1-y^{2}\right) q+2 c\right], 1\right\}
$$

where $c$ is the constant parametrizing the cohomology class. According to case 18 in table 2 , since $\mathfrak{g}_{\mathrm{c}}^{(12)}$ contains the subalgebra spanned by the vector fields $p$ and $\mathrm{e}^{-x} q$, any finite-dimensional module $n$ is spanned by functions of the form $f(x, y) \mathrm{e}^{\mu x}$, with $f(x, y)$ a polynomial in both $x$ and $y$. The second and third generators of $\mathfrak{g}_{c}^{(12)}$ map this element to

$$
f_{y} \mathrm{e}^{\{\alpha-1) x} \quad\left\{2 y f_{x}+\left(1-y^{2}\right) f_{y}+2(\mu y+c) f\right\} \mathrm{e}^{\{\mu+1) x}
$$


respectively. Taking $\mu$ to be a 'highest weight' exponent, meaning that no function of the form $g(x, y) \mathrm{e}^{(\mu+1) x}, g$ a polynomial, lies in $n$, we find that the corresponding $f$ must satisfy the differential equation

$$
2 y f_{x}+\left(1-y^{2}\right) f_{y}+2(\mu y+c) f=0 .
$$

The most general solution of this linear first-order partial differential equation is

$$
f(x, y)=(y-1)^{\mu+c}(y+1)^{\mu-c} h\left(\left(y^{2}-1\right) \mathrm{e}^{x}\right)
$$

where $h$ is an arbitrary function of its argument. In order that $f$ be a polynomial, we must have $h=$ constant and $\mu+c$ and $\mu-c$ are both non-negative integers. Therefore we obtain the quantization condition that $2 c=n$ must be an integer, and $\mu=m+n / 2$, where $m \geqslant \max \{0,-n\}$ is an integer. Then, applying the 'lowering operator' $\mathrm{e}^{-x} q$ repeatedly, we deduce that an irreducible finite-dimensional module $n_{n}^{m}$ for this Lie algebra will be spanned by the functions

$$
R_{k}^{m, n}(y) \mathrm{e}^{(m+(n / 2)-k) x} \quad k=0, \ldots, 2 m+n
$$

where

$$
R_{k}^{m, n}(y)=\frac{\mathrm{d}^{k}}{\mathrm{~d} y^{k}}\left[(y-1)^{m+n}(y+1)^{m}\right] \quad k=0, \ldots, 2 m+n .
$$

Note that $R_{k}^{m, 0}=Q_{m}^{2 m-k}$, cf (6). These polynomials can be expressed in terms of the classical Jacobi polynomials $P_{k}^{(\alpha, \beta)}$ by the formula

$$
R_{k}^{m, n}(y)=2^{k} k !(y-1)^{m+n-k}(y+1)^{m-k} P_{k}^{(m+n-k, m-k)}(y)
$$

cf [1]. In terms of the original variables, (8), the generators take the form

$$
(x-y)^{(m+(n / 2)-k)} R_{k}^{m, n}\left(\frac{x+y}{x-y}\right) .
$$

Any other finite-dimensional module for a given integer $n$ is a direct sum of the irreducible modules $n_{n}^{m}$ for a finite number of $m$ 's.

Case 16. Here, without loss of generality, the generators can be taken in the form $q$, $\xi_{i}(x) q+\eta_{i}(x, y)$, and 1 , where $i=2, \ldots, r$, and the $\eta_{i}$ are polynomials in $y$. (In order that such differential operators span a Lie algebra, there are additional conditions on the $\xi_{i}$ and the $\eta_{i}$, but this is sufficient for our purposes.) Since $q \in \mathbf{g}$, according to case 1 of table 2, any element of the associated finite-dimensional module $n$ is of the form $r(x, y) \mathrm{e}^{\mu y}$, where $r$ is a polynomial in $y$. Now applying one of the other basis operators to this function gives

$$
\left[\xi_{i} q+\eta_{i}\right] r(x, y) \mathrm{e}^{\mu y}=\left[\xi_{i} r_{y}+\left(\mu \xi_{i}+\eta_{i}\right) r\right] \mathrm{e}^{\mu \nu} .
$$

Iteration implies that, for $n$ to be finite-dimensional, we must have $\mu \xi_{i}+\eta_{i}=k_{i}$ a constant. Thus, the Lie algebra is spanned by generators of the form $q, \xi_{i}(q-\mu)+k_{i}$, and 1 , for $k_{i}, \mu$ constant, or, equivalently, $q, \xi_{i}(q-\mu), 1$. Now a simple rescaling by $\psi(x, y)=\mathrm{e}^{-\mu y}$ (see (3)), will eliminate $\mu$, and hence the Lie algebra is equivalent to one spanned by vector fields and the constant functions. (In other words, the associated cocycle is readily seen to be a coboundary, and so the cohomology must be trivial.)

Case 18. In our earlier classification, this was the most complicated case, but, fortunately, we do not need to use all of the particulars of the cohomology to determine 
the quasi-exactly solvable cases. For such a Lie algebra, the generators take the form $p$, and $\mathrm{e}^{\lambda x}(a(x) q+b(x, y))$, where the $a$ are polynomials in $x$, and the $b$ are polynomials in $x$ and $y$ (again subject to additional complicated constraints). Suppose first that the Lie algebra contains an operator of the particular form $\mathscr{D}_{0}=\mathrm{e}^{\lambda_{0} x}\left(a_{0}(x) q+b_{0}(x)\right)$. (We will prove below that there is always an equivalent Lie algebra of differential operators with this property.) Using $\mathscr{D}_{0}$, We first prove that any finite-dimensional module $n$ is spanned by functions of the form $r(x, y) \mathrm{e}^{\mu x+\nu y}$, where $r$ is a polynomial in both $x$ and $y_{0}$ Indeed, since the vector field $p$ is in $\mathrm{g}$, the module $n$ is spanned by functions $f(x, y) \mathrm{e}^{\mu x}$, where $f$ is a polynomial in $x$. Then $\mathscr{D}_{0}\left[f \mathrm{e}^{\mu x}\right]=\left(a_{0} f_{y}+b_{0} f\right) \mathrm{e}^{\left(\mu+\lambda_{0}\right) x}$. Iterating $\mathscr{D}_{0}$, and using finite-dimensionality, we deduce that for $N$ sufficiently large, if $\lambda_{0} \neq 0$, then

$$
\left(a_{0}(x) \partial_{y}+b_{0}(x)\right)^{N} f(x, y)=0
$$

while if $\lambda_{0}=0$, then $f$ must satisfy a differential equation of the form

$$
\sum_{i=0}^{N} c_{i}\left(a_{0}(x) \partial_{y}+b_{0}(x)\right)^{i} f(x, y)=0
$$

where the $c_{i}$ are constants. In either case, $f(x, y)=r(x, y) \mathrm{e}^{\nu y}$, where $r$ is a polynomial, proving the claim.

Now, let $r(x, y) \mathrm{e}^{\mu x+\nu y}$ be a fixed function in $n$, and let $\mathrm{e}^{\lambda x}(a(x) q+b(x, y))$ be any element of $g$. If $\lambda \neq 0$, the same iterative argument proves that

$$
\left[a(x) \partial_{y}+\nu a(x)+b(x, y)\right]^{N} r(x, y)=0
$$

for $N$ sufficiently large. Since $r$ and $b$ are polynomials, we deduce that $\nu a+b=0$, so the generator has the form $\mathrm{e}^{\lambda x} a(x)(q-\nu)$. On the other hand, if $\lambda=0$, then finitedimensionality implies that $r$ must satisfy a differential equation of the form

$$
\sum_{i=0}^{N} c_{i}\left[a(x) \partial_{y}+\nu a(x)+b(x, y)\right]^{i} r(x, y)=0
$$

where the $c_{i}$ are constants. Now, using the fact that $r$ and $b$ are polynomials, and equating the various powers of $y$ in (10), we first deduce that $b=b(x)$ cannot depend on $y$, and, moreover, $\nu a+b$ must be a constant. Thus, we have proved that the generators of $\mathrm{g}$ are of the form $p, \mathrm{e}^{\lambda x} a_{\lambda, j}(x)(q-\nu), \lambda \neq 0$, and $a_{0, j}(x)(q-\nu)+k_{j}$, for $\nu, k_{j}$ constant. Now the same rescaling argument as at the end of case 16 proves that the cohomology is trivial.

It remains to prove the initial claim, that, given a Lie algebra of differential operators of type 18 with $m=\{1\}$, there is an equivalent Lie algebra $\dot{\mathfrak{g}}$, which contains both the translation vector field $p$ and an operator of the form $\mathrm{e}^{\lambda_{0} x}\left(a_{0}(x) q+b_{0}(x)\right)$. We first present an elementary proof using the assumption that $g$ is quasi-exactiy soivabie. Suppose first that $\mathrm{g}$ contains a differential operator $\mathscr{D}=\mathrm{e}^{\lambda x}(q+b(x, y))$, where $b$ is a polynomial and $\lambda \neq 0$. Let $f(x, y) \mathrm{e}^{\mu x} \in n, f$ a polynomial in $x$, where $\mu$ is of 'highest weight', i.e. $n$ contains no functions of the form $g(x, y) \mathrm{e}^{(\mu+\lambda) x}$, with $g$ a polynomial in $x$. Then $\mathscr{D}\left[f(x, y) \mathrm{e}^{\mu x}\right]=\left(f_{y}+b f\right) \mathrm{e}^{(\mu+\lambda) x}$, which implies $f_{y}+b f=0$. Since $f$ is a polynomial in $x$, this is possible only if $b=b(y)$ depends on $y$ alone. But in this case, rescáling by $\psi=\overline{e x p} \int b(y) d y$, as in (3), changes $\mathscr{D}$ to the operator e $e^{\lambda x} q$ without âfecting the vector field $p$; thus we find an equivalent Lie algebra of the same canonical form containing both $p$ and $\mathrm{e}^{\lambda x} q$, as desired. The only case not covered by this argument is when every element of $\mathrm{g}$ has the form $a(x) q+b(x, y)$ for $a, b$ polynomials. In this case, $\mathbf{g}$ necessarily contains a differential operator of the form $q+\tilde{b}(x, y)$, where $\tilde{b}$ is 
a polynomial. But $[p, q+\tilde{b}]=\tilde{b}_{x}$ must lie in $m=\{1\}$, so $\tilde{b}=c x+d(y)$. As before, we can rescale to reduce the operator to $q+c x$, without affecting $p$. This completes the proof of the claim.

An alternative, but harder, proof of this fact, which has the advantage that it does not require the condition of quasi-exact solvability, can be based on a detailed analysis of the cohomology in the particular case $m=\{1\}$. In this case, the cohomology can be taken to have a particularly simple form:

Lemma 4. Let $g$ be a Lie algebra of differential operators represented by a triple $[\mathfrak{h},\{1\},[\boldsymbol{F}]]$, where $\mathfrak{h}$ is a Lie algebra vector fieid of type 18 . Then the cohomology class $[F]$ can always be represented by a ' $y$-independent cocycle' of the form

$$
p, \quad \mathrm{e}^{\lambda x}\left(x^{k} q+r_{k \lambda}(x)\right)
$$

except in the following two cases:

$$
\begin{aligned}
& p, \quad q+2 c_{1} x, \quad x q+c_{1} x^{2}+c_{2} y \\
& p, \quad \mathrm{e}^{\lambda x} q, \quad \mathrm{e}^{-\lambda x}\left(q+c_{1} y\right) \quad \text { where } \lambda \neq 0 .
\end{aligned}
$$

Proof. The proof rests on the details of the cohomology classification from $[3,4]$, and we just indicate the principal points, leaving the details to the interested reader. Using the notation there, we find that the set $\Lambda_{i, j}$ is empty for $i \neq 0, j>0$ except if $r_{0}=1$, then $\Lambda_{-1,1}=\{0\}$, while $\Lambda_{0, j}=\Lambda$ for $j>0$. (The exceptional case corresponds to the algebra (11).) Furthermore, all these exponents are linked, and hence can be absorbed by a coboundary, except if $\lambda+\mu=0, r_{\lambda}+r_{\mu}=0, j=1$, which corresponds to the second exceptional algebra (12). This completes our analysis of case 18.

Case 20. According to $[3,4]$, cf table 3 , when $m=\{1\}$, there are only two subcases where the cohomology is non-trivial:

$$
\operatorname{span}\left\{p, q+c x, x p-y q, x q+c x^{2} / 2, \ldots, x^{r} q+c x^{r} /(r+1) !, 1\right\}
$$

and

$$
\operatorname{span}\left\{p, q, x p+\frac{1}{2} y q, x q+c y, 1\right\} .
$$

In either case, using the fact that the module $n$ is spanned by functions of the form $r(x, y) \mathrm{e}^{\mu x}$, where $r$ is a polynomial in $x$, the general methods from case 18 can be similarly employed to deduce that the cohomology parameter $c$ must vanish.

Case 23. Suppose $r>2$, so that, according to table 3, the Lie algebra of differential operators is given by

$$
\mathbf{g}_{\mathrm{c}}^{(23)}=\operatorname{span}\left\{p, q, 2 x p+r y q, x q, x^{2} p+r x y q+c x, x^{2} q, \ldots, x^{r} q, 1\right\} .
$$

Moreover, since the Lie algebra of vector fields of type 20 is a subalgebra, according to table 2 , the associated module $n$ must be spanned by monomials $x^{i} y^{j}$. Now,

$$
\left[x^{2} p+r x y q+c x\right] x^{i} y^{j}=(i+r j+c) x^{i+1} y^{j} .
$$

Choosing $i$ maximal demonstrates that the cohomology must be quantized, $c=-n$, where $0 \leqslant n \in \mathbb{Z}$. For such an $n$, the corresponding module $n$ is then spanned by all monomials $x^{i} y^{j}$ for $i+r j \leqslant n$. The case $r=2$ is similar, the cohomology being initially two-dimensional, but a similar calculation proves that it reduces to the one-dimensional quantized version $c_{1}=-n, c_{2}=0$. 
This completes our sample illustrative proofs. The remaining cases in table 4 are relatively simple, or can be obtained using straightforward analogues of the arguments given above.

Finally, we remark that many of the resulting quasi-exactly solvable Lie algebras of differential operators in two complex variables are subalgebras of larger ones. It is easy to establish the following chains of inclusions for suitable values of the (quantized) cohomology parameters:

$$
\begin{aligned}
& 1 \subset 2 \subset 3 \subset 9 \subset 10 \subset 11 \quad 4 \subset 5 \subset 6 \subset 10 \subset 11 \\
& 4 \subset 5 \subset 6 \subset 8 \subset 15 \quad 4 \subset 7 \subset 8 \subset 15 \quad 12 \subset 11 \\
& 16 \subset 17 \quad 4 \subset 18 \subset 19 \quad 13 \subset 14 \subset 24 \\
& 20 \subset 22 \subset 24 \quad 21 \subset 22 \subset 24 \quad 23 \subset 24 .
\end{aligned}
$$

Thus the maximal quasi-exactly solvable Lie algebras are cases $11,15,17,19$ and 24 . (However, as remarked above, case 17 is uninteresting from the point of view of quantum mechanics as it only involves differentiation in a single direction.) This remark will serve to simplify the classification of quasi-exactly solvable Schrödinger operators in two dimensions; however, since the maximal algebras all have only trivial modules, it will still be of use to have the more detailed classification of non-maximal cases in hand, as such Schrödinger operators will have a far richer class of exact wavefunctions.

\section{Acknowledgments}

NK was supported in part by an NSERC grant, and PJO in part by NSF grant DMS 89-01600.

\section{References}

[1] Abramowitz M and Stegun I 1970 Handbook of Mathematical Functions (Washington, DC: National Bureau of Standards)

[2] Alhassid Y, Engel J and Wu J 1984 Algebraic approach to the scattering matrix Phys. Rev. Lett. 53 17-20

[3] González-López A, Kamran N and Olver P J 1991 Lie algebras of differential operators in two complex variables Am. J. Math. in press

[4] González-López A, Kamran N and Olver P J 1991 Lie algebras of first-order differential operators in two complex variables Differential Geometry, Global Analysis, and Topology (Conference Proceedings, Canadian Math. Soc.); 1991 Am. Math. Soc. in press

[5] González-López A, Kamran N and Olver P J 1991 Lie algebras of vector fields in the real plane Proc. Lond. Math. Soc. in press

[6] Jacobson N 1962 Lie Algebras (New York: Interscience)

[7] Kamran N and Olver P J 1989 Equivalence of differential operators SIAM J. Math. Anal. 20 $1172-85$

[8] Kamran N and Olver P J 1990 Lie algebras of differential operators and Lie-algebraic potentials J. Math. Anal. Appl. 145 342-56

[9] Levine R D 1988 Lie algebraic approach to molecular structure and dynamics Mathematical Frontiers in Computational Chemical Physics (IMA Volumes in Mathematics and its Applications 15) ed D G Truhlar (New York: Springer) pp 245-61 
[10] Lie S 1880 Theorie der Transformationsgruppen Math. Ann. 16 441-528; 1927 Gesammelte Abhandlungen vol 6 (Leipzig: Teubner) pp 1-94

[11] Lie S 1924 Gruppenregister Gesammelte Abhandlungen vol 5 (Leipzig: Teubner) pp 767-73

[12] Morozov A Y, Perelomov A M, Rosly A A, Shifman M A and Turbiner A V 1990 Quasi-exactly solvable quantal problems: one-dimensional analogue of rational conformal field theories Int. J. Mod. Phys. 5 803-32

[13] Shifman M A and Turbiner A V 1989 Quantal problems with partial algebraization of the spectrum Commun. Math. Phys. 126 347-65

[14] Turbiner A V 1988 Quasi-exactly solvable problems and sl(2) algebra Commun. Math. Phys. 118 467-74 